\title{
Emergence of Non albicans Candida as a Major Pathogen Isolated from Urine Samples of Patients Attending Tertiary Care Centre, Greater Noida, India
}

\author{
Shaheen Bhat, Tarana Sarwat" and Shalini Bisht \\ Department of Microbiology, School of Medical Sciences and Research, Sharda University, \\ Greater Noida U.P, India \\ *Corresponding author
}

A B S T R A C T

\begin{tabular}{|c|}
\hline Keywords \\
\hline $\begin{array}{l}\text { Antifungal } \\
\text { susceptibility, } \\
\text { Candiduria, } C \text {. } \\
\text { albicans, non albicans } \\
\text { candida }\end{array}$ \\
\hline Article Info \\
\hline $\begin{array}{l}\text { Accepted: } \\
\text { 22 July } 2018 \\
\text { Available Online: } \\
10 \text { August } 2018\end{array}$ \\
\hline
\end{tabular}

Candida albicans is the most common aetiological agent in candiduria cases commonly observed in hospitalized patients. But a few reports are available from India where non albicans Candida species have accounted for $>50$ per cent of urinary Candida isolates. We undertook this study to know the Candida species profile amongst candiduria cases. A total of 119 consecutive Candida isolates obtained from clinically suspected cases of urinary tract infection from January 2016 to June 2017 (18 months), were included. Yeast species were identified by standard phenotypic methods. Antifungal susceptibility testing of yeast was performed by disc diffusion method as per Clinical and Laboratory Standards Institute (CLSI) guidelines. The male to female ratio was 0.92. The mean age of patients was $42.7 \pm 18.9$ yr. C. tropicalis $(45.3 \%)$ was the most common Candida species followed by $C$. glabrata (22.6\%). All isolates of non-albicans Candida except few C. glabrata, were resistant to fluconazole. None of the isolates showed resistance to Amphotericin B. Non albicans Candida especially $C$. tropicalis were the predominant fungal pathogens responsible for urinary tract infection. More resistance to fluconazole observed in the study may be due to widespread use of fluconazole in this area.

\section{Introduction}

The presence of fungus in urine or candiduria is a common finding at a tertiary care hospital. Candiduria is observed in 16-22 per cent of hospitalized patients (Zarei-Mahmoudabadi et al., 2012; Kobayashi et al., 2004). Candiduria is one of the most common symptoms of urinary tract infections caused by several species of Candida, which is a normal flora of human body. Candida albicans has played an important role in candiduria (Nayman et al.,
2011). Candida species are the most common cause of fungal infections leading to a range of life threatening invasive to non-life threatening diseases (Achkar and Fries, 2010). Urinary tract infections as a result of Candida species is becoming increasingly common in hospitalised setting particularly in intensive care units (Jain et al., 2011). Epidemiological surveillance indicates that Candida species are now the most common pathogens causing nosocomial bloodstream and urinary tract infection (Horvath et al., 2003). Yeast 
belonging to the genus Candida exists as saprophytes, colonizing mucosal surfaces and external genitalia of humans of either gender, but especially near the urethralmeatus of healthy, premenopausal women. All common Candida species are capable of causing urinary tract infections (UTIs), and in many centers worldwide non-albicans Candida species now predominate (Rivett et al., 1986). Candida species accounts for almost 9 to $40 \%$ of nosocomial urinary tract infections (Achkar and Fries, 2010). About 14 Candida species have been implicated in human infections, with Candida albicans being the most prevalent among the yeast isolates. Though, the most frequently isolated species is Candida albicans, but Candida tropicalis, Candida glabrata, Candida krusei, and Candida parapsilosis are also emerging as important etiologic agents of Candida infection (Krcmery and Barnes, 2002). Reports are available where non-albicans Candida species accounted for $>50$ per cent of urinary Candida isolates (Jain et al., 2011; Singla et al., 2012). Chromagar Candida media can be reliably used for isolation of yeasts. Use of this medium even allows mycology laboratories to identify rapidly clinically important species. Chromagar Candida culture will also enable the clinician to choose appropriate antifungal drugs and thereby decreasing patients' mortality and morbidity (Horvath et al., 2003). Several reports showed that the frequency of urinary tract infection (UTI) due to yeasts has increased during the last decades (Laverdiere et al., 2007; Saha et al., 2008). Prolonged hospitalisation, long stay in ICU, urinary tract abnormality, immunocompromised patients, antibacterial therapy with broad spectrum for long time and prophylaxis by antifungal agents are presented as more important risk factors for UTI (Nayman et al., 2011; Dalen et al., 2005). A review of the epidemiology of candiduria including all retrospective reviews, case controlled studies and a large prospective surveillance study on candiduria, showed that the common risk factors include urinary tract instrumentation, prior surgical procedures, recent use of antibiotics, advanced age, female sex, diabetes mellitus, immunosuppressive therapy and prolonged hospital stay (Kobayashi et al., 2004; Cauda, 2009). The present study was aimed to isolate, speciate and perform antifungal susceptibility testing of the yeast isolates from the urine samples.

\section{Materials and Methods}

The study was conducted over a period of 18 months from January 2016 to June 2017 in a tertiary care hospital at Greater Noida, Uttar Pradesh.

\section{Inclusion criteria}

The urine samples submitted to the laboratory showing pure growth of yeast cells on repeat sampling, a significant colony count with $>10^{4}$ colony forming units/ml and direct microscopy collaborating that candiduria was present by evidence of pyuria and yeast cells were included in this study. Detailed information regarding probable risk factors like age, sex, pregnancy, diabetes mellitus, use of broad spectrum antibiotics, indwelling urinary tract catheter and presence of central venous line were recorded and included in this study.

\section{Exclusion criteria}

Urine samples which failed to grow yeast cells on repeat samples were considered contaminants and excluded from this study.

\section{Characterisation of Candida species}

Growth obtained was identified and characterized using standard techniques on the basis of Gram staining, Reynold's Braude phenomenon (Germ tube), Culture on 
CHROM agar and sugar assimilation test. Following which antifungal drug susceptibility was performed using Kirby Bauer's disc diffusion method using commercially available discs on Muller Hinton Agar with $2 \%$ glucose

Chromagar media used for isolation and speciation of Candida species

Chromagar (Himedia) was prepared according to the manufacturer's instructions. The suspension was completely dissolved by boiling

\section{Germ tube test}

Small portion of an isolated colony was suspended in a test tube containing $0.5 \mathrm{ml}$ of human serum then incubated at $37^{\circ} \mathrm{C}$ for 2 hours then examined microscopically at 30 minutes intervals up to 2 hours for the presence of germ tube.

\section{Sugar assimilation}

Five drops of Candida suspension was added to yeast nitrogen base agar after cooling at $45^{\circ} \mathrm{C}$ then poured into plates. Filter paper discs impregnated with saturated sugar solutions were placed on the surface of agar, and then incubated at $27-30^{\circ} \mathrm{C}$ up to 48 hours. Positive growth indicated by growth of Candida around the assimilated sugars.

\section{Antifungal susceptibility test}

Antifungal susceptibility testing was carried out using the disc diffusion method following the (CLSI, 2009) guidelines, using fluconazole $(25 \mu \mathrm{g})$, itraconazole $(50 \mu \mathrm{g})$, ketoconazole $(10 \mu \mathrm{g})$, and amphotericin B $(20 \mu \mathrm{g})$ antifungal discs. Supplemented Mueller-Hinton agar (Mueller-Hinton agar $+2 \%$ glucose and 0.5 $\mathrm{g} / \mathrm{mL}$ methylene blue dye, (GMB medium)) was used for performing the antifungal susceptibility testing. Preparation of inoculum: Inoculum was prepared by picking five distinct colonies of approximately $1 \mathrm{~mm}$ from 24 hours old culture grown on Saboured Dextrose Agar (SDA agar) incubated at 35$37^{\circ} \mathrm{C}$. Colonies were suspended in $5 \mathrm{ml}$ of sterile $0.85 \%$ saline. Susceptibility test procedure: Prepared plates with Mueller Hinton Agar $+2 \%$ glucose and $0.5 \mu \mathrm{g} / \mathrm{ml}$ methylene blue dye (GMB) medium for carrying out susceptibility of antifungal discs. The medium in the plates should be sterile and have a depth of about $4 \mathrm{~mm}$. The prepared inoculum streaked in the entire agar surface of the plate with the cotton swab three times, turning the plate at $60^{\circ}$ angle between each streaking. The inoculum allowed to drying for 5- 15 minutes with lid in place. The discs were applied using aseptic technique. Deposit the discs with centers at least $24 \mathrm{~mm}$ apart. Inverted the plates and placed in an incubator set to $35-37^{\circ} \mathrm{C}$ within 15 minutes after the discs were applied and examined all plate after 20-24 hours of incubation. Measured the zone diameter to the nearest whole millimeter at the point at which there is prominent reduction in growth.

\section{Results and Discussion}

Of the 119 Candida isolates, 62 (52\%) were obtained from female patients. The male to female ratio was 0.92 . The mean age was 42.7 \pm 18.9 yr. An age-wise distribution of Candiduria cases is shown in the Figure 1. Maximum cases of Candiduria were seen in 31-40 and 51-60 yr age group.

Common underlying conditions were ICU admission (17.9\%), surgical procedures $(9.8 \%)$ and diabetes mellitus (6.5\%). Pus cells per high power field observed in uncentrifuged urine is shown in the Table 1. Concomitant bacteria were also isolated in 21 (17.6\%) candidura cases. Enterococcus faecalis was isolated in $9(7.5 \%)$ cases, 
Escherichia coli in $10(8.4 \%)$ cases and Pseudomonas spp. in $2(1.6 \%)$ cases. Candida tropicalis was the most common Candida isolate $(45.5 \%)$ followed by $C$. glabrata (22.7\%), C. albicans (18.2\%) and C. krusei (13.6\%) as shown in Table 2. Germ tube test was positive in $38(29.3 \%)$ Candida isolates. All germ tube test positive isolates were $C$. albicans. Only one C. albicans isolate was germ tube negative (Fig. 2).

The antifungal sensitivity tests carried out using commercially available antifungal disc that $10(100 \%)$ strains of $C$. albicans were sensitive to amphotericin B and itraconazole, while $7(70 \%)$ strains of $C$. albicans were resistant to fluconazole and 2 (20) to Ketoconazole.

Of the C. glabrata strains, all (100\%) strains were sensitive to Itraconazole, $4(80 \%)$ were sensitive to amphotericin B and itraconazole, whereas 3 strains $(60 \%)$ were resistant to Ketoconazole. Among the C. tropicalis strains, four $(100 \%)$ were found sensitive to amphotericin B and Itraconazole.

While all four strains (100\%) were found resistant fluconazole and 2 stains resistant (20\%) to Ketoconazole. C. krusei, 3 (100\%) strains were found sensitive to amphotericin B and $100 \%$ resistant to Fluconazole and Ketoconazole, followed by $1(33.3 \%)$ to Itraconazole antifungal agents. Amphotericin B and itraconazole $21(95.5 \%)$ was found to be the most effective antifungal agent (Table 3 and 4; Figure 3).

Nosocomial candidial UTI is fast gaining an important place in tertiary care hospitals. The significance of the presence of yeasts in urine of patients is not clearly understood (Nucci, 2000). A common clinical problem is deciding whether candiduria represents urinary tract infections or merely bladder colonization or contamination. Distinguishing contamination from true infection is not easy, despite the existence of reliable diagnostic criteria for significant candiduria. However, candiduria is sometimes a marker of disseminated candidiasis (Sobel, 2002). A total of 119 Candida isolates from urine clinical specimens were included in this study, of which C.tropicalis showed the highest number of isolates $(45.3 \%)$, followed by C.glabrata (22.6\%), C. albicans (17.6\%) and C. krusei $(14.2 \%)$ respectively.

According to Patel et al., Candida species is the seventh most common nosocomial hospital wise pathogen, which caused $25 \%$ of all the urinary tract infections (Patel et al., 2012). Other studies have documented that hospitalised patients are relatively susceptible to candiduria (Kobayashi et al., 2004; Sellami et al., 2006).

The majority of candiduria in the present study were caused by non albicans species, especially C. tropicalis (45.3\%), emerging as a nosocomial infection. $C$. albicans had remained the major agents of candiduria until recently, however; several reports show that non albicans species, especially $C$. tropicalis and $C$. glabrata now predominate in many regions (Weinberger et al., 2003).

Similar fungal profile has been reported by other studies from India (Jain et al., 2011; Paul et al., 2007; Paul et al., 2004). Our study observed that females were affected predominantly $(52 \%)$, contrary to the male predominance reported in the study by (Paul et al., 2007).

Several reports show that the frequency of candiduria in women is more than men (Achkar and Fries, 2010). In this study most of the candiduria cases were adults $(76.3 \%$ were 21-60 yr of age). There were two peaks of candiduria cases: one at 31-40 yr and another was 51-60 yr. Elderly people are at higher risk of candiduria because of decreased immunity in advance age (Jain et al., 2011). 
Table.1 Microscopic observation of pus cells in candiduria cases (n-119)

\begin{tabular}{|c|c|}
\hline $\begin{array}{l}\text { Pus cells per high power field in } \\
\text { uncentrifuged urine }\end{array}$ & $\begin{array}{l}\text { No. (\%) of } \\
\text { urine samples }\end{array}$ \\
\hline Nil & $79(66.4 \%)$ \\
\hline Upto 5 & $13(10.9 \%)$ \\
\hline $6-10$ & $4(3.4 \%)$ \\
\hline $11-15$ & $6(5.1 \%)$ \\
\hline $16-20$ & $7(5.8 \%)$ \\
\hline $21-25$ & $2(1.6 \%)$ \\
\hline$>25$ & $8(6.7 \%)$ \\
\hline
\end{tabular}

Table.2 Gender wise distribution of Candida species isolated from urine samples

\begin{tabular}{|l|l|l|l|}
\hline Species & $\begin{array}{l}\text { Number of isolates } \\
(\%)\end{array}$ & Male & Female \\
\hline C.tropicalis & $54(45.3 \%)$ & 19 & 35 \\
\hline C. glabrata & $27(22.6 \%)$ & 10 & 17 \\
\hline C. albicans & $21(17.6 \%)$ & 15 & 6 \\
\hline C. krusei & $17(14.2 \%)$ & 13 & 4 \\
\hline Total & 119 & 57 & 62
\end{tabular}

Table.3 Number (\%) of C. albicans to Non albicans Candida isolates

\begin{tabular}{|l|l|}
\hline C. albicans & Non albicans Candida \\
\hline $21(\mathbf{1 7 . 6 \%})$ & $98(82.3 \%)$ \\
\hline
\end{tabular}

Table.4 Antifungal susceptibility pattern of Candida species causing UTIs

\begin{tabular}{|c|c|c|c|c|}
\hline Species & 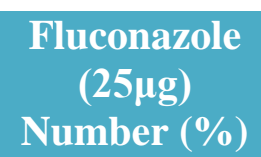 & 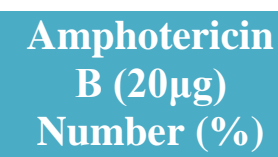 & $\begin{array}{c}\text { Itraconazole } \\
\quad(50 \mu \mathrm{g}) \\
\text { Number }(\%)\end{array}$ & $\begin{array}{c}\text { Ketoconazole } \\
(10 \mu g) \\
\text { Number }(\%)\end{array}$ \\
\hline $\begin{array}{l}\text { C.tropicalis } \\
(\mathrm{n}-54)\end{array}$ & $0(0.0 \%)$ & $54(100 \%)$ & $54(100 \%)$ & $0(0.0 \%)$ \\
\hline $\begin{array}{l}\text { C.glabrata } \\
(\mathrm{n}-27)\end{array}$ & $6(22 \%)$ & $27(100 \%)$ & $23(85 \%)$ & $11(40.7 \%)$ \\
\hline $\begin{array}{l}\text { C.albicans } \\
(\mathrm{n}-21)\end{array}$ & $7(33.3 \%)$ & $21(100 \%)$ & $21(100 \%)$ & $5(23.8 \%)$ \\
\hline $\begin{array}{l}\text { C.krusei } \\
\text { (n-17) }\end{array}$ & $0(0.0 \%)$ & $17(100 \%)$ & $11(64.7 \%)$ & $0(0.0 \%)$ \\
\hline
\end{tabular}


Fig.1 Age-wise distribution of candiduria cases

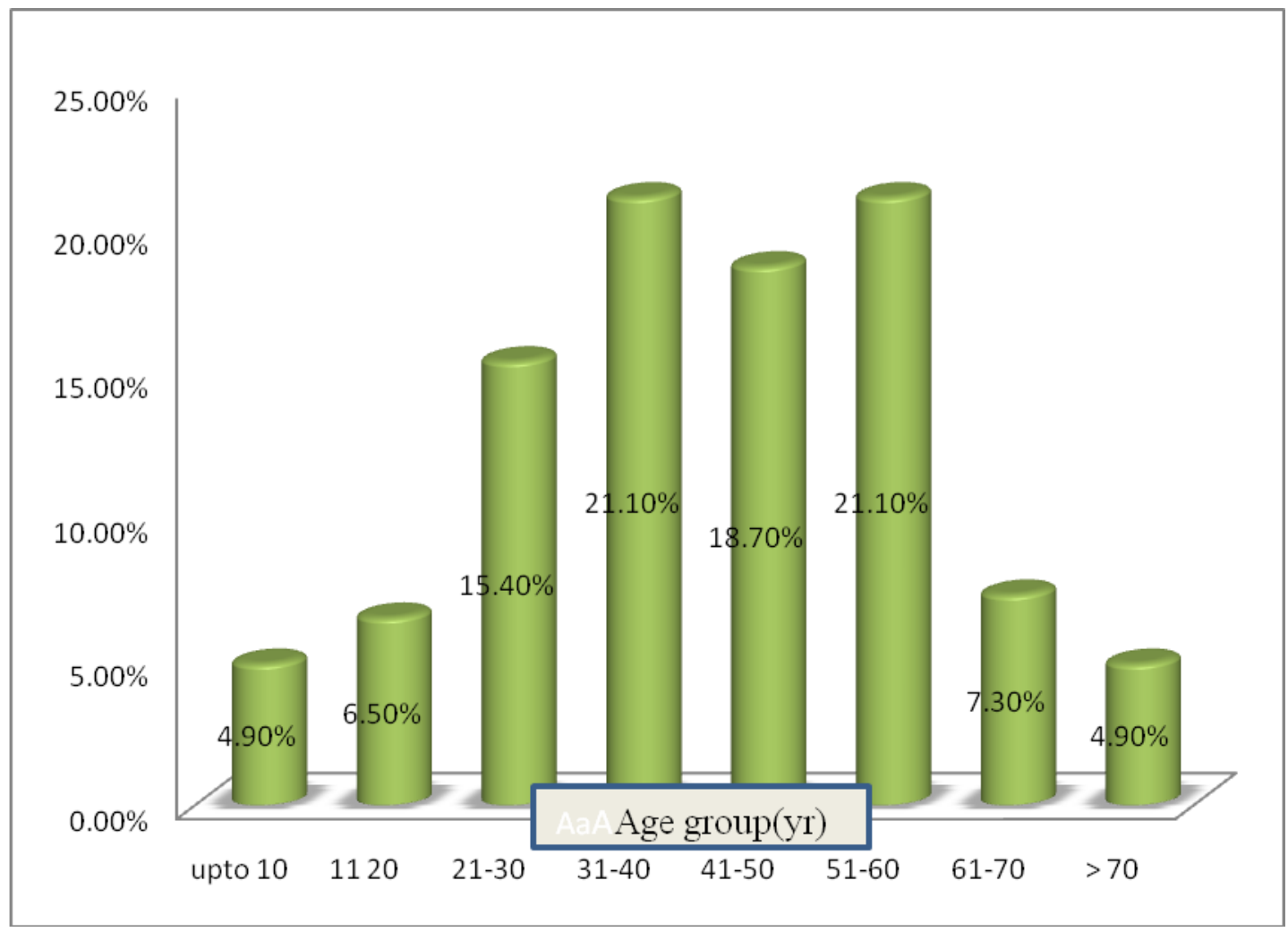

Fig.2 Percentage of C.albicans to Non albicans Candida isolates from Urine samples

C.albicans,

$17.60 \%$

Non albicans

candida, $82.30 \%$ 
Fig.3 Antifungal sensitivity pattern of Candida species isolated from UTIs

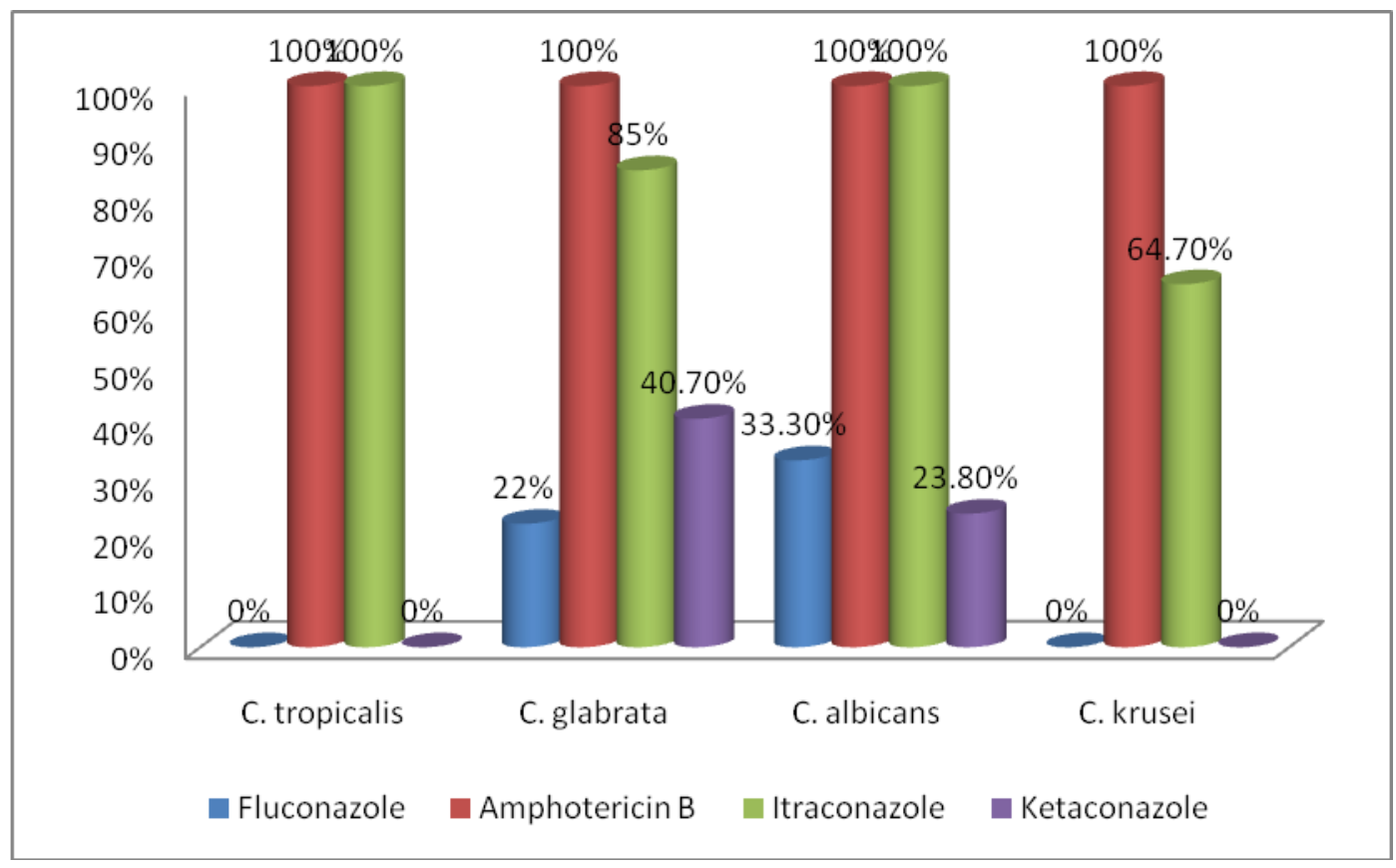

Pyuria ( $>5$ pus cells/high-power field) was observed only in 22.6 percent of candiduria cases. Hence, candiduria was not associated with the presence of pus cells in urine in our study. Presence of pus cell in candiduria cases may be due to coexistent bacterial infection or mechanical injury of the bladder mucosa by indwelling urinary catheter. Bacterial coinfection was observed in 17.6 per cent of candiduria cases in the current study. The present study shows that all isolates of nonalbicans Candida i.e., C. tropicalis, C. krusei and $C$. glabrata were resistant to fluconazole, with the exception of 6 isolates of $C$. glabrata that were sensitive to fluconazole. Amphotericin B and itraconazole were found to be the most effective antifungal agent. UTIs due to $C$. glabrata have recently increased and these infections are usually resistant to fluconazole (Yang et al., 2003). The susceptibility range of Candida species varies to antifungal drugs. C. albicans are usually sensitive to amphotericine B. However, several reports show that nonalbicans are more resistant to antifungal, especially fluconazole and believe that the differences in sensitivity pattern of Candida species to antifungal are associated with geographical distributions (Saha et al., 2008; Yang et al., 2008).

Based on the present study it is emphasised that there is the need of considering candiduria as an emerging and important entity in today's scenario. The presence of candiduria represents therapeutic challenge for physician and should be verified by the second clean catch urine culture. The susceptibility of yeasts to antifungal agents cannot always be predicted and therefore testing individual yeast pathogens against the appropriate antifungal agents is often necessary. Antifungal susceptibility testing in vitro ensures that the drug that will be chosen will be active against the infecting organism and therefore provide beneficial therapeutic effect to the patient under treatment. Since our study indicates the upcoming resistance of Candida species to the antifungal agents in use hence it is of utmost importance not only to identify Candida up to species level but also to conduct its antifungal profile. 


\section{References}

Achkar JM, and Fries BC. Candida infections of the genitourinary tract. Clinical microbiology reviews. 2010 Apr 1; 23(2): 253-73.

Achkar JM, and Fries BC. Candida infections of the genitourinary tract. Clinical microbiology reviews. 2010 Apr 1; 23(2): 253-73.

Cauda R. Candidaemia in patients with an inserted medical device. Drugs. 2009 Nov 1; 69(1): 33-8.

Clinical and Laboratory Standards Institute (CLSI). Methods for antifungal disc diffusion susceptibility testing of yeasts; approved guideline, 2nd ed. CLSI document M44-a2: CLSI; 2009.

Dalen DM, Zvonar RK, Jessamine PG. An evaluation of the management of asymptomatic catheter-associated bacteriuria and candiduria at The Ottawa Hospital. Canadian Journal of Infectious Diseases and Medical Microbiology. 2005; 16(3): 166-70.

Horvath LL, Hospenthal DR, Murray CK, Dooley DP. Direct isolation of Candida spp. from blood cultures on the chromogenic medium CHROMagar Candida. Journal of clinical microbiology. 2003 Jun 1; 41(6): 262932.

Jain M, Dogra V, Mishra B, Thakur A, Loomba PS, Bhargava A. Candiduria in catheterized intensive care unit patients: emerging microbiological trends. Indian journal of pathology and microbiology. $2011 \mathrm{Jul} \mathrm{1;} \mathrm{54(3):} \mathrm{552-55.}$

Kobayashi CC, Fernandes OD, Miranda KC, de Sousa ED, Silva MD. Candiduria in hospital patients: a study prospective. Mycopathologia. 2004 Aug 1; 158(1): 49-52.

Kobayashi CC, Fernandes OD, Miranda KC, de Sousa ED, Silva MD. Candiduria in hospital patients: a study prospective.
Mycopathologia. 2004 Aug 1; 158(1): 49-52.

Krcmery V, and Barnes AJ. Non-albicans Candida spp. causing fungaemia: pathogenicity and antifungal resistance. Journal of Hospital Infection. 2002 Apr 1; 50(4): 243-60.

Laverdiere M, Labbé AC, Restieri C, Rotstein C, Heyland D, Madger S, Stewart T. Susceptibility patterns of Candida species recovered from Canadian intensive care units. Journal of critical care. 2007 Sep 1; 22(3): 245-50.

Nayman SA, Özguneș I, Ertem OT, Erben N, Doyuk EK, Tözun M, Usluer G. Evaluation of risk factors in patients with candiduria. Mikrobiyolojibulteni. 2011 Apr; 45(2): 318-24.

Nucci M. Candiduria in hospitalized patients: a review. The Brazilian journal of infectious diseases: an official publication of the Brazilian Society of Infectious Diseases. 2000 Aug; 4(4): 168-72.

Patel LR, Pethani JD, Bhatia P, Rathod SD, Shah PD. Prevalence of Candida infection and its antifungal susceptibility pattern in tertiary care hospital, Ahmedabad. Natl J Med Res. 2012; 2(4): 439-1.

Paul N, Mathai E, Abraham O, Michael JS, Mathai D. Factors associated with candiduria and related mortality. Journal of Infection. 2007 Nov 1; 55(5): 450-5.

Paul N, Mathai E, Abraham OC, Mathai D. Emerging microbiological trends in candiduria. Clinical infectious diseases. 2004 Dec 1; 39(11): 1743-4.

Rivett AG, Perry JA, Cohen J. Urinary candidiasis: a prospective study in hospital patients. Urological research. 1986 Sep 1; 14(4): 183-6.

Saha R, Das SD, Kumar A, Kaur IR. Pattern of Candida isolates in hospitalized 
children. The Indian Journal of Pediatrics. 2008 Aug 1; 75(8): 858-60.

Sellami A, Sellami H, Makni F, Bahloul M, Cheikh-Rouhou F, Bouaziz M, Ayadi A. Candiduria in intensive care unit: significance and value of yeast numeration in urine. In Annales francaisesd'anesthesieet de reanimation 2006 Jun (Vol. 25, No. 6, pp. 584-588).

Singla N, Gulati N, Kaistha N, Chander J. Candida colonization in urine samples of ICU patients: determination of etiology, antifungal susceptibility testing and evaluation of associated risk factors. Mycopathologia. 2012 Aug 1; 174(2): 149-55.

Sobel JD. Controversies in the diagnosis of candiduria: what is the critical colony count. Curr Treat Opt Infect Dis. 2002; 4: 81-3.

Weinberger M, Sweet S, Leibovici L, Pitlik SD, Samra Z. Correlation between candiduria and departmental antibiotic use. Journal of Hospital Infection. 2003 Mar 1; 53(3): 183-6.

Yang YL, Cheng HH, Ho YA, Hsiao CF, Lo HJ. Fluconazole resistance rate of Candida species from different regions and hospital types in Taiwan. Journal of microbiology, immunology, and infection $=$ Wei mianyugan ran zazhi. 2003 Sep; 36(3): 187-91.

Yang YL, Wang AH, Wang CW, Cheng WT, Li SY, Lo HJ. Susceptibilities to amphotericin B and fluconazole of Candida species in Taiwan Surveillance of Antimicrobial Resistance of Yeasts 2006. Diagnostic microbiology and infectious disease. 2008 Jun 1; 61(2): 175-80.

Zarei-Mahmoudabadi A, Zarrin M, Ghanatir F, Vazirianzadeh B. Candiduria in hospitalized patients in teaching hospitals of Ahvaz. Iranian journal of microbiology. 2012 Dec; 4(4): 198.

\section{How to cite this article:}

Shaheen Bhat, Tarana Sarwat and Shalini Bisht. 2018. Emergence of Non albicans Candida as a Major Pathogen Isolated from Urine Samples of Patients Attending Tertiary Care Centre, Greater Noida, India. Int.J.Curr.Microbiol.App.Sci. 7(08): 3956-3964. doi: https://doi.org/10.20546/ijcmas.2018.708.408 